\title{
Studies of the Magnetization Reversal Processes in Co Dot and Antidot Arrays on a Microscopic Scale
}

\author{
M. Vázquez ${ }^{*}$, A. Asenjo ${ }^{1}$, W. O. Rosa ${ }^{1,2}$, I. Mínguez ${ }^{1}$, M. Hernández-Vélez ${ }^{3}$, M.-Y. $\operatorname{Im}^{4}$ and P. Fischer ${ }^{4}$ \\ ${ }^{1}$ Instituto de Ciencia de Materiales de Madrid, CSIC, 28049, Madrid, Spain \\ ${ }^{2}$ Universidad de Oviedo, 33005, Oviedo, Spain \\ ${ }^{3}$ Universidad Autónoma de Madrid, Cantoblanco, 29049, Madrid, Spain \\ ${ }^{4}$ Center for X-ray Optics, Lawrence Berkeley National Lab., Berkeley CA 94720, USA
}

\begin{abstract}
Co dot and antidot arrays with hexagonal symmetry have been prepared by combined electrochemical and sputtering techniques with significantly reduced nanoscale dimensions. Their magnetization reversal process has been analyzed on a macroscopic scale with Vibrating Sample Magnetometry (VSM) and on a microscopic scale by high spatial resolution Magnetic Transmission Soft X-ray Microscopy (MTXM). We observe for the antidot arrays a nucleation and propagation mode driven reversal mechanism induced by the in-plane anisotropy starting with nucleated clusters following the hexagonal configuration, and the magnetic hardness (i.e., coercivity) depending on the geometry parameters. Contrary, for arrays of short dots, a significant longitudinal anisotropy is deduced with large coercivity, where magnetostatic interactions gives rise to a more complex reversal process and significantly reduced remanence and susceptibility.
\end{abstract}

Keywords: Magnetization reversal, hexagonal symmetry self-ordering, ferromagnetic dot and antidot arrays, magnetic transmission soft X-ray microscopy.

\section{INTRODUCTION}

Recently, advances in novel generations of magnetic recording media of enhanced storage density and magnetic logic $[1,2]$ have triggered increased interest in the study of magnetic behavior and particularly in the reversal process of networks of magnetic nanoelements. Arrays of dots and antidots (porous thin films) are among the most interesting alternatives due to their great potential in storage capacity where each dot or domain wall pinning centre in antidot arrays can be considered as a single bit [3, 4].

Such arrays are generally synthesized by various lithography techniques. Typical periodicities in the range of 400-500 $\mathrm{nm}$ with square or hexagonal symmetry arrangement and circular, square or triangular shaped dots and antidots with lateral dimensions down to around $200 \mathrm{~nm}$ have been achieved [5-7]. Alternative methods include an electrochemical route to prepare magnetic nanowires by filling self-assembled pores in anodic alumina membranes [8-10], or magnetic porous thin films (arrays of antidots) by sputtering onto such membranes [11-13]. In this case, selfordering processes allow for hexagonal symmetry with periodicity down to around $65 \mathrm{~nm}$, and pore diameter of 20 to $40 \mathrm{~nm}$.

A fundamental understanding of the magnetization reversal mechanisms in these magnetic dot and antidot arrays on a microscopic length scale is scientifically very

\footnotetext{
*Address correspondence to this author at the Instituto de Ciencia de Materiales de Madrid, CSIC, 28049, Madrid, Spain; Tel: +34 913349051; Fax:+34 913720623; E-mail: mvazquez@icmm.csic.es
}

interesting, but moreover mandatory to determine their actual potentials for technological applications. In fact, geometrical characteristics play a dominant role here. Three main types of magnetic configurations occur for cylindrical elements depending on the length-to-diameter ratio of the individual dots. This ratio determines e.g. whether the magnetic configuration exhibits in-plane or out-of-plane magnetization easy axis, or whether a vortex-like magnetic structure forms [14]. Particularly, it includes the knowledge of intrinsic magnetization reversal mechanism of individual dots/antidots as well as of the role of existing magnetostatic interactions [15].

So far, to characterize such systems experimentally, most studies have been performed by Superconducting Quantum Interference Devices, SQUID, or Vibrating Sample Magnetometry, VSM, which provide macroscopic information on the magnetization reversal process averaged over a macroscopic ensemble of dots or antidots arrays. However, theoretical models suggest the impact of magnetic interactions considering dipolar or more complex magnetostatic coupling among such elements on a microscopic scale $[16,17]$. Alternative techniques include MOKE measurements on a periodically perforated films [18], but particularly magnetic imaging techniques with high spatial resolution, e.g., magnetic force microscopy, MFM, provide microscopic, i.e. local information of magnetization reversal processes of nanostructured samples [19, 20]. Unfortunately, for soft magnetic materials such as Co dots and antidots the stray field of the MFM probes interacting with the specimen is disadvantageous and yields quite often ambiguous results [21]. This can be overcome by Magnetic Transmission Soft X-ray Microscopy, MTXM [22, 23], 
which is one of the most advanced and powerful techniques to experimentally visualize and quantitatively determine insitu magnetization processes. Element specific magnetic contrast is provided by $\mathrm{X}$-ray magnetic circular dichroism, $\mathrm{XMCD}$, utilizing the strong dependence in X-ray absorption on the relative orientation between photon helicity and the projection of the local magnetization component along the propagation direction of the X-rays. Since the X-ray images can be recorded in varying external magnetic fields, MTXM can be used as quantitative and local magnetometry tool [24].

In the present work, we have focused our study on the magnetization reversal process of Co dot and antidot arrays with particularly small-scale geometry characteristics. We have combined MTXM imaging and VSM magnetometry to provide complementary microscopic and macroscopically averaged information, respectively. From the analysis of data supplied by both techniques, we conclude similar magnetization processes which are largely determined by the particular magnetic anisotropy distribution of each sample.

\section{SAMPLE PREPARATION}

A hexagonal ordering of the pore arrays is achieved by self-assembling during double anodization of the aluminum substrates [8-10]. Thus, an anodic porous alumina membrane with pore diameter of $35 \mathrm{~nm}$ and lattice parameter of $105 \mathrm{~nm}$ is obtained. To match the limited penetration depth of soft $\mathrm{X}$-rays the alumina membrane thickness was kept well below $1 \mu \mathrm{m}$. With the attenuation length of soft X-rays at the Co L3 edge $(778 \mathrm{eV})$ being $0.53 \mu \mathrm{m}$, MTXM studies in those systems are very challenging in terms of signal/noise ratio.

Optimizing the preparation parameters was mandatory for a controlled fabrication of dot arrays by filling the pores via electroplating. Particularly, the oxide barrier at the bottom of the anodic alumina pores was reduced down to 5 $\mathrm{nm}$ by using voltage steps. Then, the pores were filled up to reach homogeneous Co dots $35 \mathrm{~nm}$ in diameter and a very short height of $150 \mathrm{~nm}$. The width of the height distribution is about $15 \%$. We want to note, that the short wires (dots) are buried under the membrane since its height is much lower than the length of the alumina pores. In the following such an array of Co dots is labeled as D150.

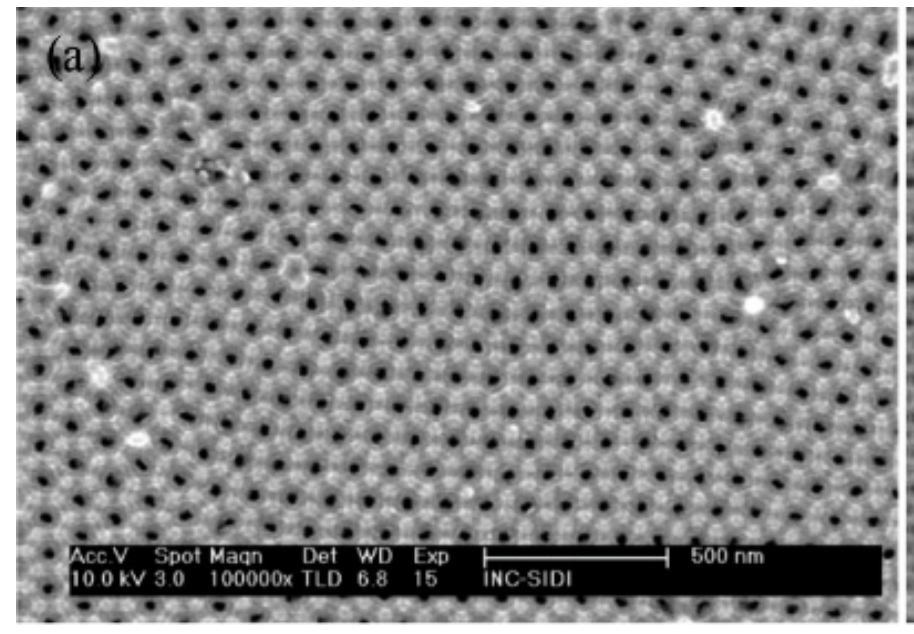

On the other hand, antidot arrays were obtained by sputtering deposition of Co onto anodic alumina used as substrate [25]. The thickness of investigated arrays was 30 and $50 \mathrm{~nm}$, samples which are labeled as AD30 and AD50, respectively. After the deposition, a $2 \mathrm{~nm}$ thick $\mathrm{Cu}$ capping layer was deposited to protect the film. It should be mentioned that upon sputtering a small magnetic mass enters the alumina pores and reduces the diameter of antidots [11, 26]. Final diameters of antidots were about $35 \mathrm{~nm}$ and $32 \mathrm{~nm}$ for $\mathrm{AD} 30$ and $\mathrm{AD} 50$, respectively.

The actual periodicities of the Co arrays as well as the dot and antidot diameters were determined from careful analysis of Scanning Electron Microscopy (SEM) imaging. In all cases, a narrow diameter distribution was achieved, with a variation of $\pm 2 \mathrm{~nm}$. As an example, Fig. (1) shows the hexagonal ordering of both types of samples.

\section{STUDIES OF MAGNETIZATION PROCESSES}

Magnetic full field transmission soft X-ray microscope at the beamline 6.1.2 at Advanced Light Source in Berkeley, CA using circularly polarized X-rays emitted off-orbit from a bending magnet was utilized to image the magnetic structures of the arrays throughout their hysteresis cycles. Standard Fresnel zone plates used for this experiment provided a spatial resolution of about $25 \mathrm{~nm}$. Since the magnetic contrast scales with the scalar product between photon helicity and sample magnetization it is possible to image either in-plane (antidots) or out-of-plane (nanodots) magnetization by tilting the specimen at an axis perpendicular to the X-ray beam. Magnetic fields up to 1 $\mathrm{kOe}$ with the field direction pointing either along the plane or out-of-plane were applied during the image recording to follow the magnetization reversal process. Magnetic domain imaging is obtained by tuning the X-ray energy to the Co L3-edge $(778 \mathrm{eV})$ thereby utilizing the strong X-ray magnetic circular dichroism effect around the Co absorption edge. To enhance the magnetic contrast and to reduce nonmagnetic background, every image corresponding to a given applied magnetic field is normalized to a reference image taken under saturating magnetic field. Particularly, WSxM free software commonly devoted to analyze SPM images has been used to improve the MTXM contrast. Since the XMCD effect constitutes a direct measure of the local element-

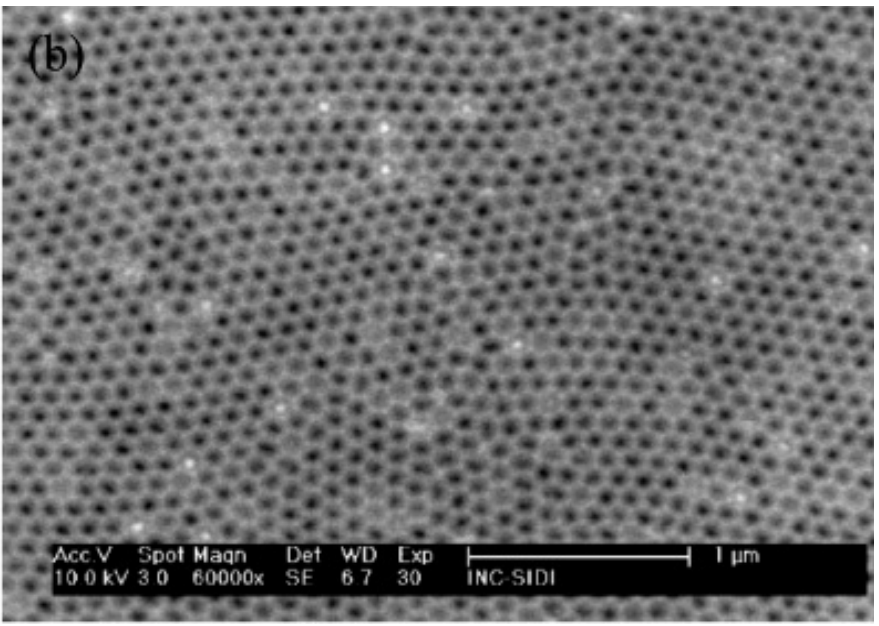

Fig. (1). SEM images of Co AD50 antidot sample (a) and dot arrays (b) reproducing the pores self-ordering of precursor anodic alumina. 
specific magnetization, an analysis of the MTXM images allowed us to deduce element specific hysteresis loops in the various samples.

\subsection{Magnetic Antidots}

The magnetization reversal process of antidot arrays has been studied by VSM and MTXM analysis. The normalized magnetization value under a given in-plane applied magnetic field is obtained by quantitative analysis of the contrast in the MTXM images. Fig. (2) shows the in-plane hysteresis loops for AD30 and AD50 Co antidot arrays derived from VSM (a) and VSM and MTXM (b) measurements. A inplane anisotropy can be assumed in both samples as deduced from the high remanence to saturated magnetization ratio. The small differences in the magnetization values between MTXM and VSM loops at identical values of applied magnetic fields are indicative of variations of magnetization in local regions (10 micrometer diameter) that can be identified by MTXM imaging, while the VSM data represent an average over the whole sample.

MTXM images of the local domain structure recorded at selected applied fields are additionally shown in Fig. (3). Circular images $(10 \mu \mathrm{m}$ in diameter) show the domain configuration of the sample at certain magnetic fields. With the chosen polarization of the X-rays, the magnetic images exhibit bright contrast after saturating under a positive magnetic field. With decreasing magnetic field, dark contrast appears in some regions indicating the reversal of the magnetic moment locally. Domains containing a given amount of antidots are nucleated with preferred orientation parallel to the applied field in the sample plane and locally, the domain walls go along the interhole lines, following the hexagonal ordering. These regions widen with increasing field strength, which suggests that the low-field magnetization process is irreversibly driven by multiple nucleation and propagation of domain walls.

In very good agreement with VSM measurement, the analysis of the MTXM images confirms in the AD30 sample a switching field of around 100 Oe. Nevertheless, quantitative magnetization data to reproduce the full hysteresis loop are difficult to obtain due to the weak magnetic contrast associated with the small amount of the magnetic component (Co) in the sample. Hence, only VSM data are presented in Fig. (2a). In turn, for the AD50 sample, the stronger contrast allows to determine a full hysteresis loop from the MTXM images by evaluating the XMCD contrast of the images. Again, in good agreement with VSM data, a coercivity of 130 Oe is found.

A comparative analysis of the shape of hysteresis loops and MTXM images for both the AD30 and the AD50 samples indicates that the overall magnetization processes are similar. Magnetization reversal occurs seemingly by domain wall motion generated at low fields below around 200 Oe indicating a moderate soft magnetic character. At higher fields magnetization approaches saturation by nearlyreversible mechanism. However, some difference can be observed. Particularly, a magnetically softer behavior is deduced for the AD30 sample, with slightly lower coercivity.

A comparison of the domain sizes indicated that the domains in the AD50 are significantly smaller than for AD30 under identical applied magnetic fields. For instance, the domain size near the coercive field in AD30 and AD50 samples is about $130 \mathrm{~nm}$ and $70 \mathrm{~nm}$, respectively. In addition, the density of nucleation of reversed domains in the AD50 sample is about a factor two higher than for the AD30 sample. That indicates that the energy required to nucleate walls in the AD30 is higher than in the AD50 sample or alternatively, that the wall energy density is stronger reduced for the sample AD50. That indicates a stronger in-plane magnetic anisotropy in the AD30 sample. However, the slightly higher in-plane coercivity and smaller remanence observed for AD50 is consistent with the existence of an outof-plane anisotropy component.
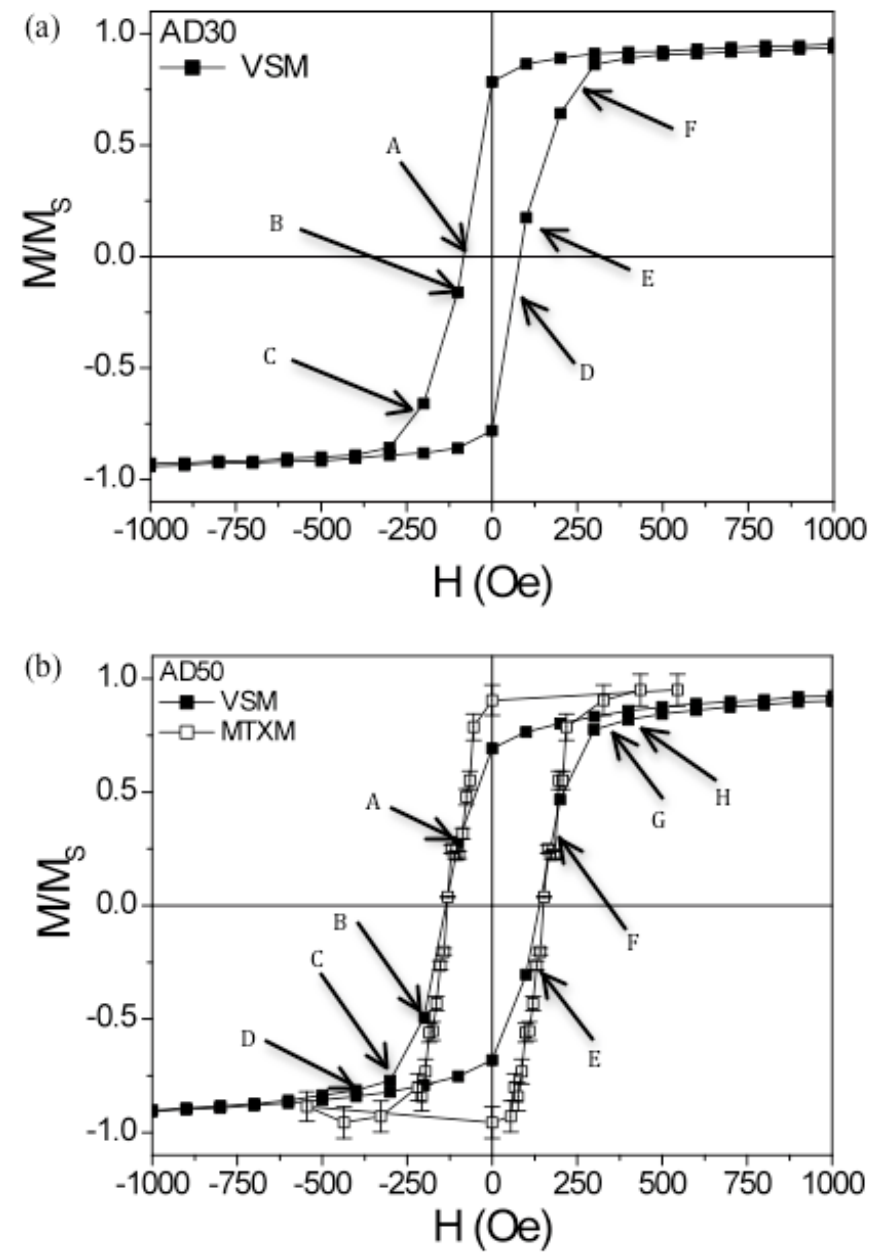

Fig. (2). In-plane hysteresis loops of AD30 (a) and AD50 (b) samples determined by MTXM and VSM.

The modest differences of magnetic behavior in the two samples can be explained by the particular sample geometries. As measured from SEM images the final diameters of holes in both AD30 and AD50 are very similar (less than $10 \%$ difference). However, the AD50 sample is significantly thicker than AD30 sample so that, its in-plane shape magnetic anisotropy is expected to be smaller.

In order to study the magnetization process in more detail, we present the changes in the magnetic moment distribution between two values of magnetic field applied in the horizontal direction of the images. Fig. (4) shows clearly individual antidots which are hexagonally arranged. The bright contrast in Fig. (4a) -marked in red in Fig (4c)- and the corresponding dark contrast in Fig. (4b) -marked in red 
in Fig (4d)- denotes regions where magnetization has reversed magnetization under the given applied field increment.
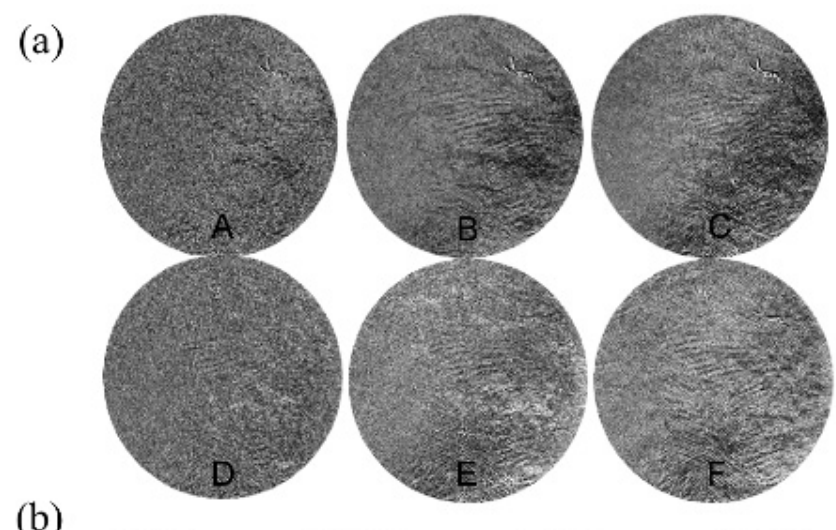

(b)

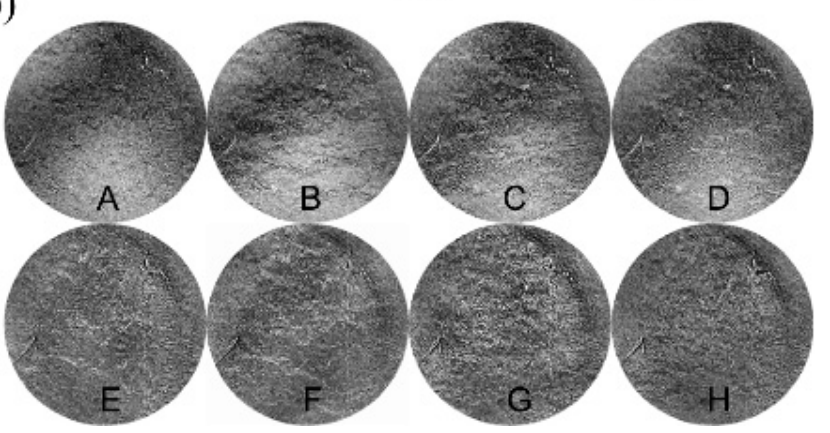

Fig. (3). MTXM series of images corresponding to AD30 (a) and AD50 (b) samples obtained at representative selected applied fields marked in Fig. (2). The magnetic field is applied in the horizontal direction of the images. Diameter of the images is $10 \mu \mathrm{m}$.

For AD30 sample, the image shows the change when the magnetic field varies from 200 Oe to 220 Oe. We observe that local regions where magnetization reverses extend along nearly the orientation of the applied field, and correlate with the local hexagonal arrangement of the antidots, which points to the relationship with the antidot pinning effect. In the same way, the discrete increment of magnetic field in sample AD50 (from -290 Oe to -310 Oe) allows us to visualize the detailed reversal process. In this latter case, the movement of the walls again correlates with the morphology

(a)

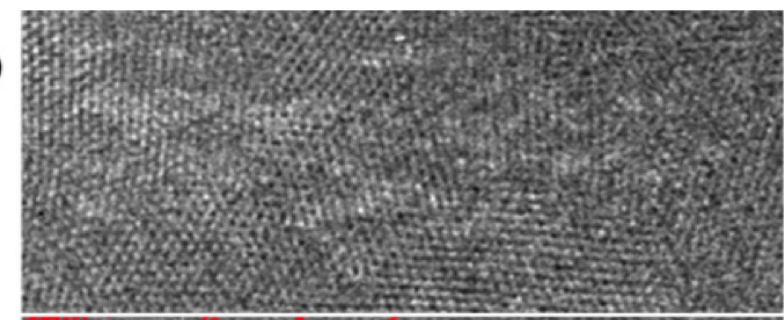

(c)

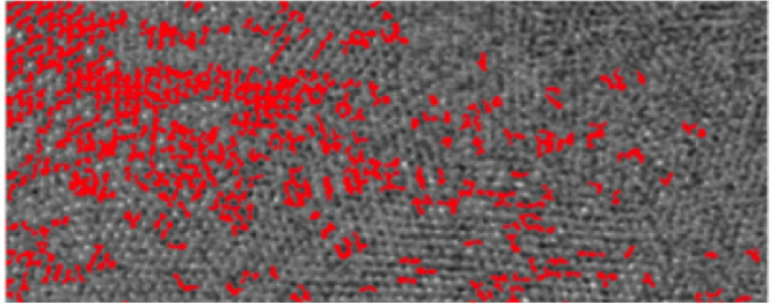

of the nanoporous magnetic film, i.e., pinning effects can be deduced.

\subsection{Magnetic Dots}

Fig. (5a) shows the out-of-plane (parallel to dots axis) VSM hysteresis loop and the corresponding MTXM magnetic domain images for the D150 Co dot array. The inplane VSM hysteresis loop (not shown here) shows a much more reduced susceptibility, and a larger anisotropy field of around 3300 Oe. A comparative analysis of these loops confirms the existence of a net out-of-plane anisotropy, with an effective magnetization easy axis parallel to the nanodot axis.

That conclusion confirms data very recently reported for arrays of short Co nanowires [27]. X-ray diffraction patterns of short Co nanowires (i.e., up to about $300 \mathrm{~nm}$ in length), prepared in off-equilibrium electrodeposition, show a major presence of $f c c$ crystallites [28]. The balance between magnetocrystalline anisotropy of that polycrystalline structure and the longitudinal shape anisotropy generates a resultant magnetization easy axis parallel to the wires axis with a coercive field of 1300 Oe. It should be mentioned that arrays of long Co nanowires (several $\mu \mathrm{m}$ in length) exhibit mainly $h c p$ crystalline structure with $c$ axis oriented nearly transversely to the wires axis [29-31]. Therefore, in that case transverse magnetocrystalline anisotropy nearly compensates the axial shape anisotropy and results in a reduced coercivity (i.e., typically in the order of a few hundreds of Oe).

On the other hand and in spite of such effective longitudinal anisotropy, the hysteresis loop in Fig. (5a) shows relatively reduced remanence which is ascribed to the noticeable role played by the magnetostatic interactions among neighboring nanodots.

A more detailed image of the magnetization process can be visualized in Fig. (5b). The dark contrast regions show the magnetization reversal process between two magnetic fields values (3250 Oe and 4250 Oe). Note that several clusters of nanowires reverse simultaneously and randomly as can also be observed in Fig. (5c) an (5d). Such images reminds one of the complex structures observed in previous study that denote the presence of relevant magnetic dipolar-

(b)

(d)

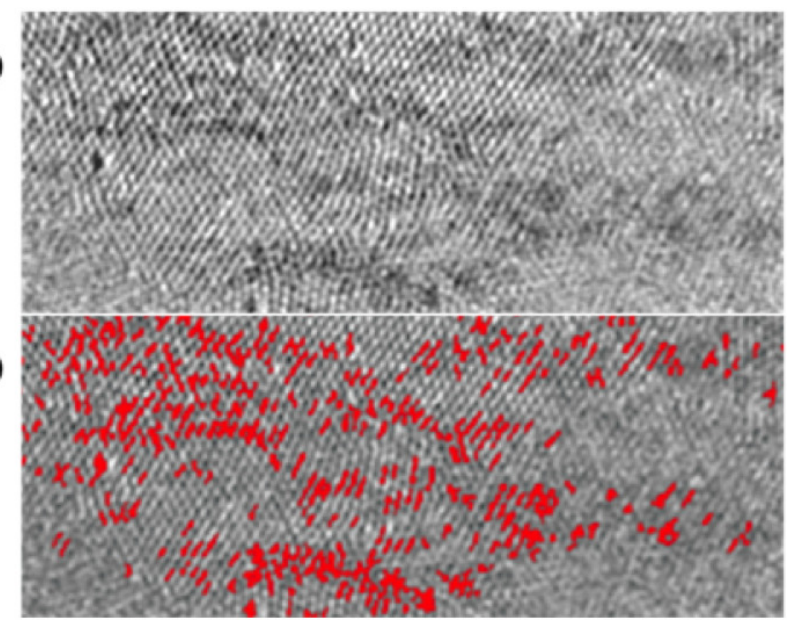

Fig. (4). Magnetization reversal process in AD30 (a and c) and AD50 (b and d) samples. The images correspond to the changes in the magnetic configuration when the field applied along the $\mathrm{X}$ direction changes as indicated in the text. The size of the images is $5 \mu \mathrm{m} \times 2,3 \mu \mathrm{m}$. 
like interactions [32].

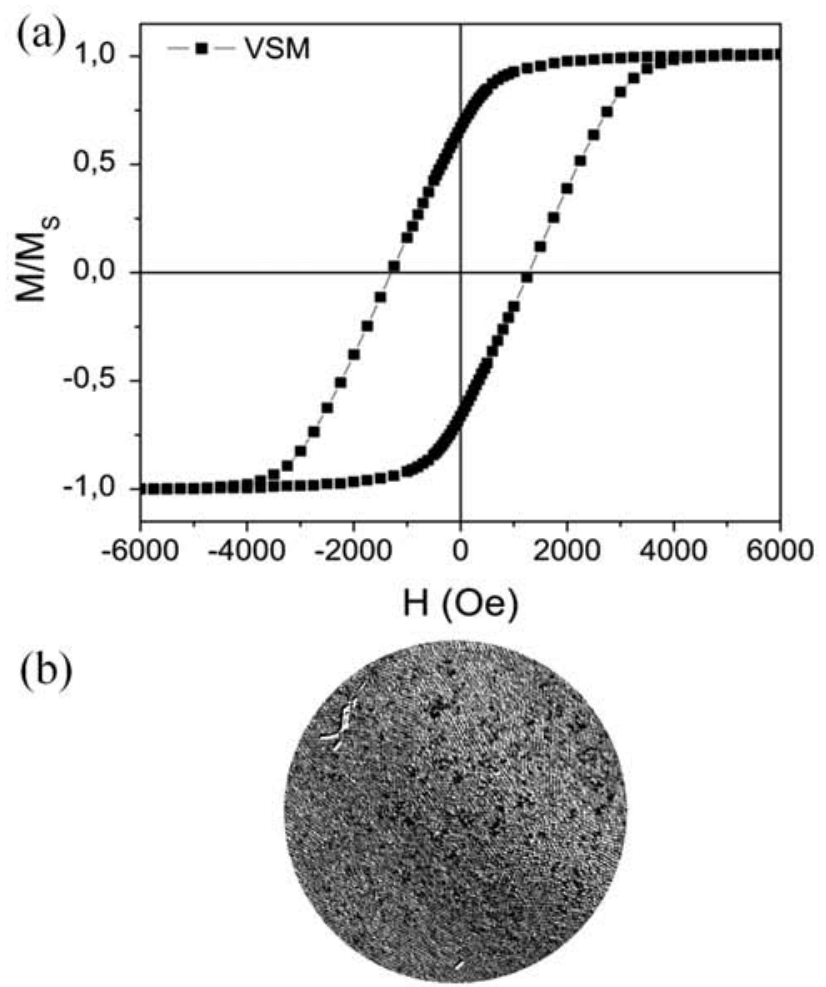

(c)

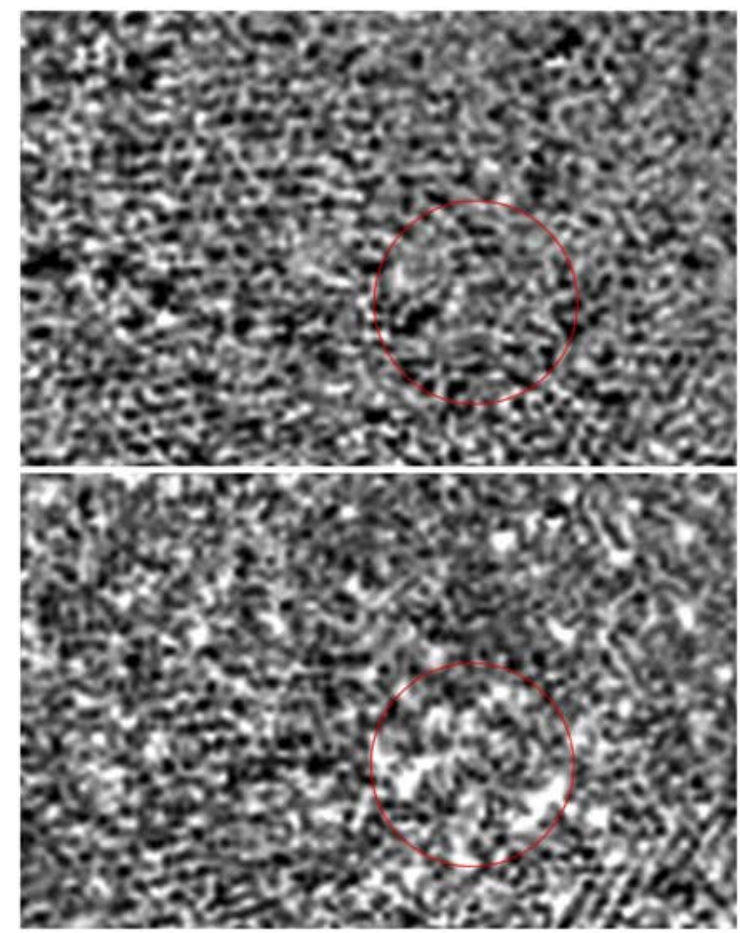

Fig. (5). (a) Out-of-plane VSM hysteresis loop for the D150 sample. (b) MTXM image ( $8 \mu \mathrm{m}$ in diameter) corresponding to the change in the magnetization configuration between 3250 Oe and 4250 Oe magnetic fields. (c) and (d) MTXM images corresponding to the magnetic configurations at $-1000 \mathrm{Oe}$ and $+1000 \mathrm{Oe}$, the size of the images is $3,2 \mu \mathrm{m} \times 2 \mu \mathrm{m}$. Red circles are drawn to locate the same regions of the sample in both images.
According to micromagnetic simulations $[14,33]$, magnetic anisotropy with a easy axis along the nanopillar is expected owing to the ratio of length to diameter in dots. That should yield a squared out-of-plane hysteresis loop with remanence close to saturation magnetization, and a coercivity of about $2 \mathrm{kOe}$ given by the switching field required to propagate a domain wall [34]. In the present case, in addition to the mentioned axial anisotropy, magnetostatic interactions are expected to occur [17], since the separation among dots is of the same order as their diameter and length. As has been previously shown, such interactions are reflected in reduction of low-field susceptibility, and remanence.

\section{CONCLUSIONS}

In summary, we have investigated the magnetization reversal process of Co antidot and dot arrays with low-scale geometry. Analysis of magnetic soft X-ray microscopy providing a spatial resolution of $25 \mathrm{~nm}$ allows us to visualize local reversal of magnetization and obtain local (microscopic) hysteresis loops whereas VSM magnetometry provides complementary (macroscopic) and laterally averaged loops of the samples.

Detailed analysis of MTXM images allows us to directly observe the evolution of the overall magnetic domain structure under stepped changes of the applied field. An inplane magnetic anisotropy is deduced for the antidot arrays with moderate differences in the studied samples. Images under different applied field correlate well with the hexagonal ordering imposed by the template and indicate a magnetization process which is governed by nucleation and propagation domain wall mechanisms. Arrays of very short Co dots exhibit a longitudinal magnetic anisotropy with large axial coercivity, where magnetic interactions play a noticeable role to reduce remanence.

\section{ACKNOWLEDGEMENT}

Authors are deeply indebted to Dr. R. Sanz for his strong support in the samples preparation. W.O. Rosa acknowledges the Brazilian Government Agency CAPES for the financial support. The work has been supported by the Spanish Ministry under project MAT2010-20798-C05-01 and by the Director, Office of Science, Office of Basic Energy Sciences, Materials Sciences and Engineering Division, of the U.S. Department of Energy under Contract No. DE-AC02-05-CH11231.

\section{CONFLICT OF INTEREST}

The authors confirm that this article content has no conflicts of interest.

\section{REFERENCES}

[1] Martin JI, Nogues J, Liu K, Vicent JL, Schuller IK. Ordered magnetic nanostructures: fabrication and properties. J Magn Magn Mater 2003; 256: 449-501

[2] Allwood DA, Xiong G, Faulkner CC, Atkinson D, Petit D, Cowburn RP. Magnetic domain-wall logic. Science 2005; 309: 1688-92.

[3] Pirota KR, Knobel M, Hernández-Vélez M, Nielsch K, Vázquez M. Magnetic nanowires: fabrication and characterization. In: Narlikar AV, Fu YY, Eds. Handbook of nanoscience and nanotechnology. USA: Oxford University Press 2010; pp. 772-824.

[4] Heyderman LJ, Nolting F, Backes D, et al. Magnetization reversal in Co Antidots. Phys Rev B 2006; 73: 214429. 
[5] Ross CA. Patterned Magnetic Recording Media. Ann Rev Mat Sci 2001; 31: 203-35.

[6] Castaño FJ, Nielsch K, Ross CA, Robinson JWA, Krishnan R. Anisotropy and magnetotransport in ordered magnetic antidot arrays. Appl Phys Lett 2004; 85: 2872-4.

[7] Wang CC, Adeyeye AO, Singh N. Magnetic antidot nanostructures: effect of lattice geometry. Nanotechnology 2006; 17: 162936.

[8] Masuda H, Fukuda K. Ordered metal nanohole arrays made by a two-step replication of honeycomb structures of anodic alumina. Science $1995 ; 268$ : 1466-8.

[9] Nielsch K, Müller F, Li AP, Gösele U. Uniform nickel deposition into ordered alumina pores by pulsed electrodeposition. Adv Mater 2000; $12: 582-6$

[10] Vázquez $\mathrm{M}$, Hernandez-Velez $\mathrm{M}$, Pirota $\mathrm{K}$, et al. Arrays of $\mathrm{Ni}$ nanowires in alumina membranes: magnetic properties and spatial ordering. Eur Phys J B 2004; 40: 489-97.

[11] Xiao ZL, Han CY, Welp U, et al. Nickel antidot arrays on anodic alumina substrates. Appl Phys Lett 2002; 81: 2869-3.

[12] Lei Y, Chim W-K, Zhang Z, et al. Ordered nanoporous nickel films and their magnetic properties. Chem Phys Lett 2003; 380: 313-8.

[13] Rahman MT, Dumas RK, Eibagi N, et al. Controlling magnetization reversal in $\mathrm{Co} / \mathrm{Pt}$ nanostructures with perpendicular anisotropy. Appl Phys Lett 2009; 94: 042507.

[14] d'Albuquerque e Castro J, Altbir D, Retamal JC, Vargas P. Scaling approach to the magnetic phase diagram of nanosized systems. Phys Rev Lett 2002; 88: 237202-4.

[15] Encinas-Oropesa A, Demand M, Piraux L, Huynen I, Ebels U. Dipolar interactions in arrays of nickel nanowires studied by ferromagnetic resonance. Phys Rev B 2001; 63: 104415.

[16] Kartopu G, Yalçın O, Es-Souni M, Başaran AC. Magnetization behavior of ordered and high density Co nanowire arrays with varying aspect ratio. J Appl Phys 2008; 103: 093915-6.

[17] Vázquez M, Nielsch K, Vargas P, et al. Modelling hysteresis of interacting nanowires arrays. Physica B Condensed Matter 2004; 343: 395-402.

[18] Torrado JF, Papaioannou ETh, Ctistis G, et al. Plasmon induced modification of the transverse magneto-optical response in $\mathrm{Fe}$ antidot arrays. Physica Status Solidi RRL 2010; 4: 271-3.

[19] Jaafar M, Yanes R, Asenjo A, et al. Field induced vortex dynamics in magnetic Ni nanotriangles. Nanotechnology 2008; 19: 285717-8.

[20] Jaafar M, Gómez-Herrero J, Gil A, Ares P, Vázquez M, Asenjo A. Variable-field magnetic force microscopy. Ultramicroscopy 2009; 109: 693-9.
[21] Meier G, Eiselt R, Bolte M, Barthelmess M, Eimüller T, Fischer P. Comparative study of magnetization reversal in isolated and strayfield coupled microcontacts. Appl Phys Lett 2004; 85. 1193-5.

[22] Fischer P, Eimüller T, Schütz G, et al. Element-specific imaging of magnetic domains at $25 \mathrm{~nm}$ spatial resolution using soft $\mathrm{x}$-ray microscopy. Rev Sci Instrum 2001; 72: 2322-4.

[23] Fischer P, Kim D-H, Chao W, Liddle JA, Anderson EH, Attwood DT. Soft X-Ray microscopy of nanomagnetism. Mater Today 2006; 9: 26-33.

[24] Im M.-Y, Fischer P, Eimüller T, Denbeaux G, Shin S-C. Magnetization reversal study of $\mathrm{CoCrPt}$ alloy thin films on a nanogranular-length scale using magnetic transmission soft $\mathrm{x}$-ray microscopy. Appl Phys Lett 2003; 83: 4589-91.

[25] Vázquez M, Pirota KR, Navas D, et al. Ordered magnetic nanohole and antidot arrays prepared through replication from anodic alumina templates. J Magn Magn Mater 2008; 320: 1978-83.

[26] Pirota KR, Prieto P, Neto AMJ, Sanz JM, Knobel M, Vázquez M. Coercive field behavior of permalloy antidot arrays based on selfassembled template fabrication. J Magn Magn Mater 2008; 320: e235-8.

[27] Vivas LG, Yanes $R$, Chubykalo-Fesenko $O$, Vázquez $M$. Coercivity of ordered arrays of Co nanowires with controlled variable length. Appl Phys Lett 2011; 98: 232507.

[28] Pirota KR, Vazquez M. Arrays of electroplated multilayered $\mathrm{Co} / \mathrm{Cu}$ nanowires with controlled magnetic anisotropy. Adv Eng Mater 2005; 7: 1111-3

[29] Liu Z, Chang P-C, Chang C-C, Galaktionov E, Bergmann G, Lu JG. Shape anisotropy and magnetization modulation in hexagonal cobalt nanowires. Adv Funct Mater 2008; 18: 1573-8.

[30] García JM, Asenjo A, Velazquez J, et al. Magnetic behavior of an array of cobalt nanowires. J Appl Phys 1999; 85: 5480-2.

[31] Kröll M, Blau WJ, Grandjean D, et al. Magnetic properties of ferromagnetic nanowires embedded in nanoporous alumina membranes. J Magn Magn Mater 2002; 249: 241-5.

[32] Escrig J, Altbir D, Jaafar M, Navas D, Asenjo A, Vázquez M. Remanence of $\mathrm{Ni}$ nanowire arrays: influence of size and labyrinth magnetic structure. Phys Rev B 2007; 75: 184429.

[33] Guslienko KY, Metlov KL. Evolution and stability of a magnetic vortex in a small cylindrical ferromagnetic particle under applied field. Phys Rev B 2001; 63: 100403(R).

[34] Sellmyer DJ, Zheng M, Skomski R. Magnetism of Fe, Co and Ni nanowires in self-assembled arrays. J Phys Condens Matter 2001; 13: R433-R60. 